\title{
AN INTELLIGENT DATA-DRIVEN ANALYTICS System To Assist SPORTS Player Training AND IMPROVEMENT USING INTERNET-OF- THINGS (IOT) AND BIg DATA ANALYSIS
}

\author{
Julius $\mathrm{Wu}^{1}$, Jerry Wang ${ }^{2}$, Jonathan Sahagun ${ }^{3}$ and $\mathrm{Yu} \mathrm{Sun}^{4}$ \\ ${ }^{1}$ Irvine High School, Irvine, USA \\ ${ }^{2}$ SMIC Private School, Shanghai, China \\ ${ }^{3}$ California State University, Los Angeles, CA, 91706 \\ ${ }^{4}$ California State Polytechnic University, Pomona, CA, 91768
}

\begin{abstract}
Our product is a very unique tracking tool that not only tracks the movement of players on a map, but also the velocity of each player. We have an application that coaches usually hold onto during a game or a practice. It shows coachesan accurate data sample of where each player is and what they are doing on the field whether it be grinding or fooling around. It also helps coaches see accurate gameplay during a game if the recording is not available. When coaches select elite players, they also get a presentation of each players' skills and how accurate they are when running different routes.
\end{abstract}

\section{KEYWORDS}

IoT, Machine learning, Data Mining.

\section{INTRODUCTION}

The application we develop is to track football players' data like numbers of steps, average velocity when training and use this data to provide summary and recommendation for football players. We expect this program will be loved by many American football players around the world as this program provides useful tips for them [1]. Compared to other traditional ways of training, our program has several benefits. First, the cost of training using this application is relatively low. We only need a kit that has an accelerator, GPS, and cellular network to track your data and report it back to your application. Second, football players can get access to detailed information through your application that can't be found in the traditional way. On the application, football players can not only see their average velocity and steps they run during matches, but also the trajectory of matches and the graph relating velocity and time. From these data, the application can automatically give a summary of your performance and possibly a suggestion for your training. Combined with detailed information, football players can have customized training, save time, and maximize their performance in big matches [2]. This application also has low barriers and it's universal to many football players, whether professional or not.

Existing method and tool use mobile phones to track the steps and locations that we need for the football tracker. This method seems good and low cost, while there's several problems within it. 
The first problem is the accuracy. Mobile phones usually have dozens of meters' variation to the actual location. This inaccuracy will cause inaccurate positioning and has a very negative impact on sportsmen's training. People will not know the close speed they run during the game and can hardly improve their training effect. The second problem is that we need to keep the mobile screen on in order to gain accurate locations. Many smartphones have a power-saving mechanism that makes applications stop tracking if the screens go off [3]. As soccer players play their sports, they don't care about their phones so their screens will inevitably go out. When the screen goes off, the application will stop tracking users' location, steps, etc. Thus, when the game is over, the athlete can't get an accurate result for their games.

Our goal in this research paper is to present their speed, location, and trajectory plot so they can use them to improve their training effect. Our method is inspired by the increasing iot networks around the world. These iot networks can connect items like bicycles together. After you ride that bicycle, the mobile phone will automatically show some basic information like speed, distance you traveled, and the map which shows you trajectory. These features are provided by dedicated logic boards. We believe that this dedicated logic board has advantages in several aspects. First, it can track athletes' information all the time without disconnecting like a mobile phone. Football games usually last about one and half hours, perhaps more. By using our tool kit to track location and speed information, athletes will not worry about being unable to collect information because it's always connected to the cloud through cellular network and sends you speed and location [4]. In this way, athletes can focus only on the game instead of checking their phones to assure that it's connected to the cloud. The second benefit is that this kit provides more accurate information thana mobile phone. Compared to mobile phones, the kit provides us more accurate location information. Typically, the error of locations of mobile phones can be up to dozens of meters. On the other hand, this kit provides you the error of location as small as several meters. The smaller the error, the more accurate the location is. If we have a more accurate location, we can track the athletes locations more precisely thus can present a more accurate trajectory plot. The kit can also count steps more accurately since it carries professional sensors [5]. The more accurate steps count can provide a more accurate distance and average speed values. With all this more accurate information, athletes can use this information to improve their strategy to play football games.

In two application scenarios, we demonstrate how the above combination of techniques increases the accuracy of tracking players' speed and location [6]. First, we show the usefulness of our approach: a case study in which one player carries the device and crosses the line by 50 times. The device will detect whether the player crosses the line or not. The player will run at a variety of speeds to make the result we test more authentic. In the second experiment, we will do the same set of tests on 5 different players. These players will carry our devices and cross the line. They will also run at a variety of speeds. Each player will cross the line for 10 times. The purpose is to test whether different players will affect the accuracy of our device to track speed and location.

The rest of the paper is organized as follows: Section 2 gives the details on the challenges that we met during the experiment and designing the sample; Section 3 focuses on the details of our solutions corresponding to the challenges that we mentioned in Section 2; Section 4 presents the relevant details about the experiment we did, following by presenting the related work in Section 5. Finally, Section 6 gives the conclusion remarks, as well as pointing out the future work of this project. 


\section{Challenges}

In order to build the tracking system, a few challenges have been identified as follows.

\subsection{How to integrate the hardware with software at real-time}

The first challenge we encountered is to learn how the programming software works. As we only had a little foundation about programming, we need to learn how the android studio works [7]. The first problem is basically installing the software. As it is a development tool, the android studio offers us with a variety of customization options. We need to figure out what plug-in we need for this software. During this process, the software runs errors so we need to spend time using Windows command processor (CMD) to solve the problem in order to let it run [8]. The second problem is learning the programming language. As we know, different programming software relies on different languages. The language we rely on is Dart. In order to program our app, we need to learn the Dart language. We need to learn proper terms, arrangements, and logic. Studying this language takes us time since we not only need to know these terms but also need to operate and try them to make them familiar and make the program work.

\subsection{How to debug and update the system program remotely}

When we write our program, we will inevitably encounter problems. In these months we wrote the applications, this happened many times. I remembered that when we wrote the login function, we couldn't log in despite finding nothing wrong with our program written to support this function and the firebase which authenticates the username and the password. This problem puzzled us for quite a long time as we inspected the code android studio and information in the firebase again and again. After checking it over and over again, we find out that it's actually the problem of connecting firebase to android studio. Although codes in android studio and the user information in firebase had setted up seperately, it's not connected with one another. It means that when we input username and password in android studio, it can't check with the firebase, thus we can't login it. To solve this problem, we inserted a firebase sdk to let the cloud connect with the program so that the problem of login and registering is solved. Following that, we can build more features like map, tracking information, etc.

\subsection{How to collect and persist information at large-scale}

In the beginning, we thought something that we could just do was to zoom in on the maps enough to scope in on a field and have our data collected there, so we did not think much further than that. Later while we were testing, we could see the tracker collect the data on a larger scale but as soon as we zoomed in on the map, everything got blurred out. We later realized that due to some restrictions, zooming in too much on a map would blur out everything rendering the field unclear. With that in the way, we could not see our players on the map clearly and we couldn't tell where on the field they would be at. We solved this problem by projecting an imaginary field onto the map by plugging in the coordinates of 2 opposite corners of the football field onto the map. With the field correctly positioned on the map, we could now graph out where players would be at.

\section{SOLUTiON}

The football tracking program shows trajectory map, duration, distance, average speed, and start and end time. The program is designed to visualize the performance of athletes in games by using data. By analyzing the data combined with the trajectory map, athletes can find out their problemsand make customized plans for their training in order to perform better in the next game or next season [9]. 
The football tracking application works with three parts: the application, the cloud(firebase), and the lot kit. The lot kit basically collects critical information we need for the application. The information includes distance, duration time, locations tracked by GPS sensor. These locations are then sent to the firebase from the device using cellular network. Thisinformation will be sent to the corresponding account. If we open the application and log in the corresponding account, we can see data we collected. By clicking different dates, we can see information we collected like distance, duration time, and locations. By using this information, the application created a trajectory map that let you see the trajectory during the game.

This system is implemented with the following components:

1) Mobile app development

2) Server/Firebase

3) Sensors and hardware

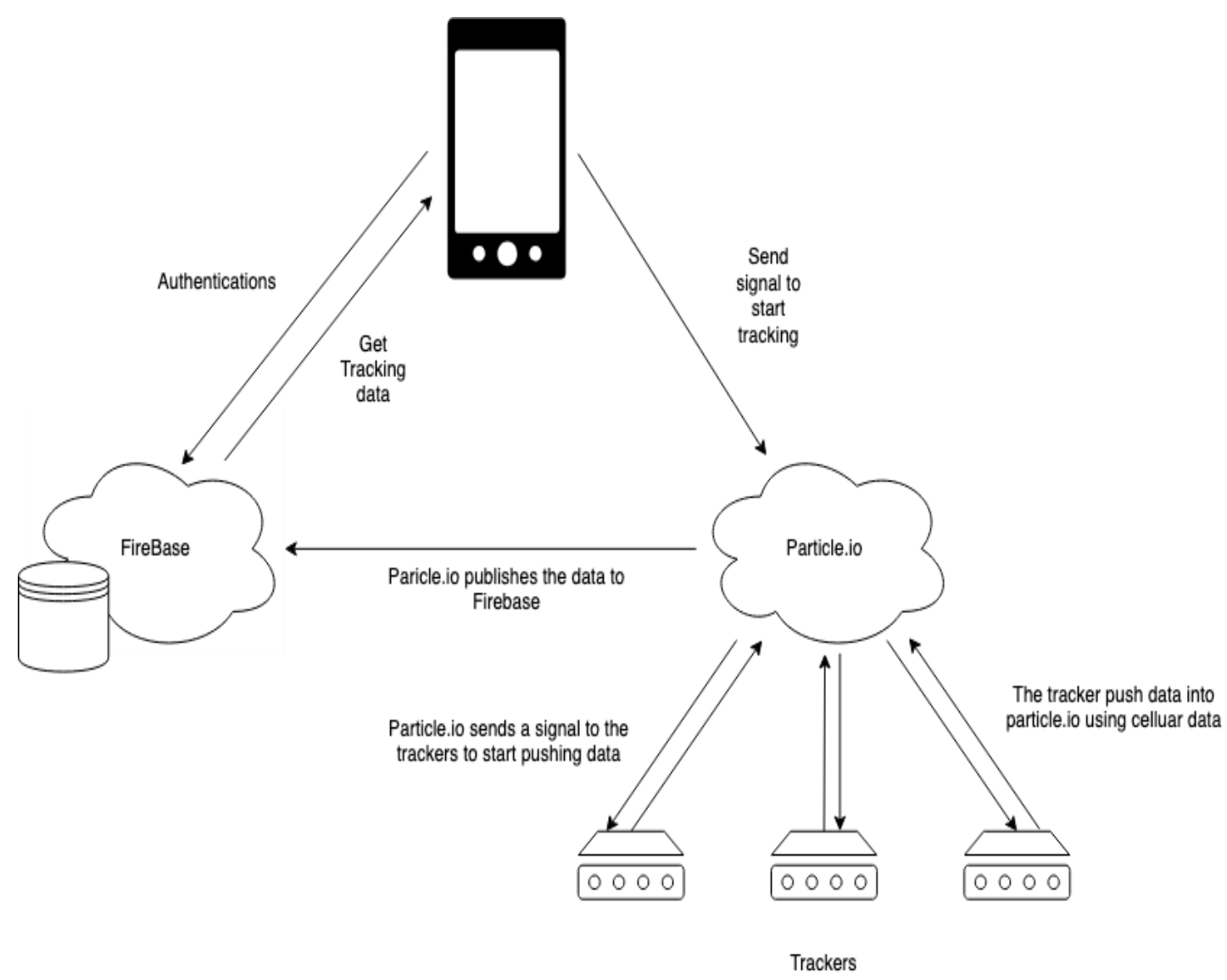

Figure 1. The overview of the solution 
Computer Science \& Information Technology (CS \& IT)

\begin{tabular}{l}
$2: 18$ Football Tracking \\
\hline $2021-6-7$ 8:37:51 \\
\hline $2021-6-5$ 20:6:32 \\
\hline $2021-6-5$ 19:31:19 \\
\hline
\end{tabular}

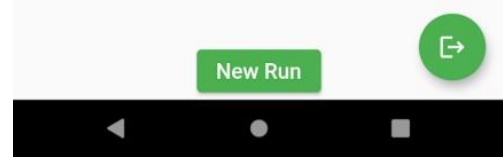

Figure 2. List of dates the tracker was running
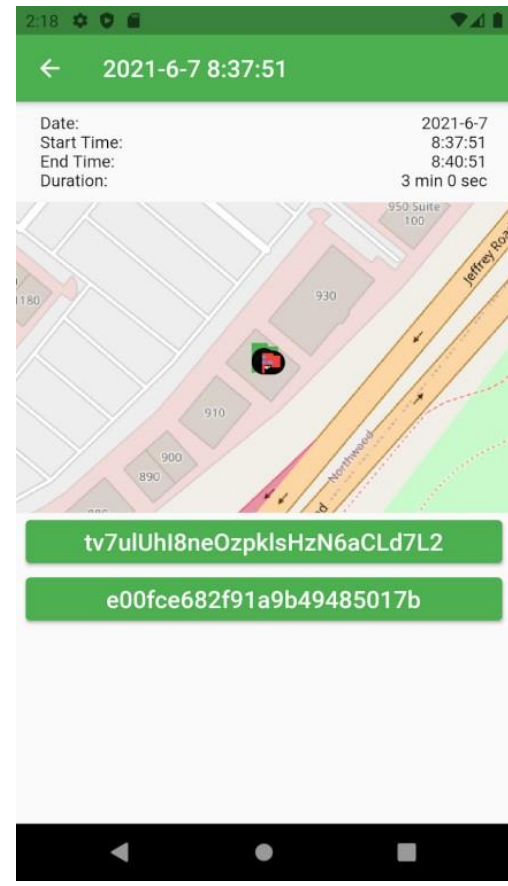

Figure 3. Example of one the dates 


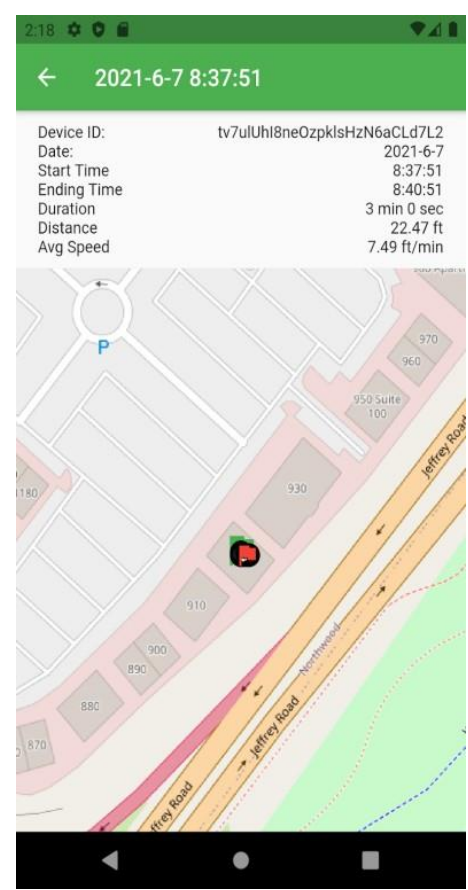

Figure 4. Example of one of the trackers details

This function sends a message to the particle.io to tell the device to start publishing

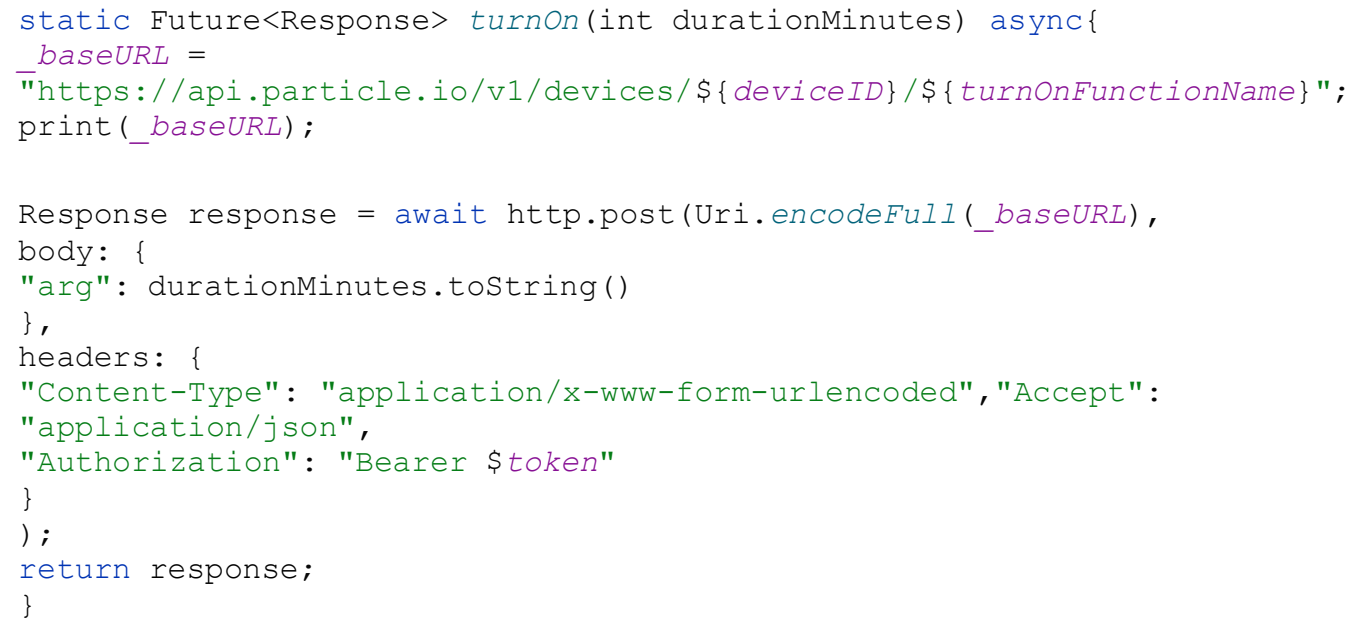

This function sends a message to the particle.io to tell the device to stop publishing. 


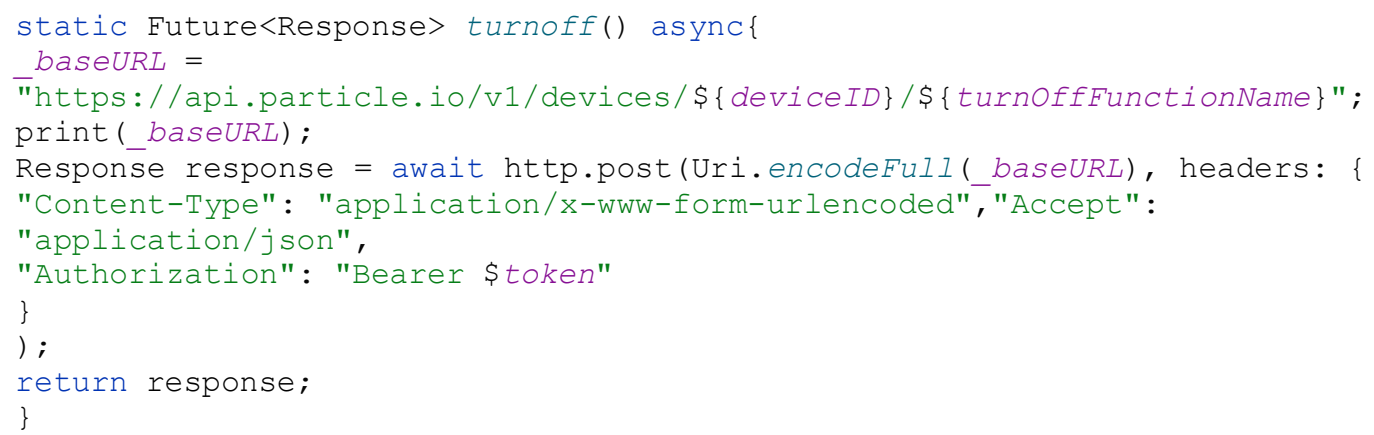

The next three functions calculate the speed of a player/device by its GPS coordinates and its timestamps.

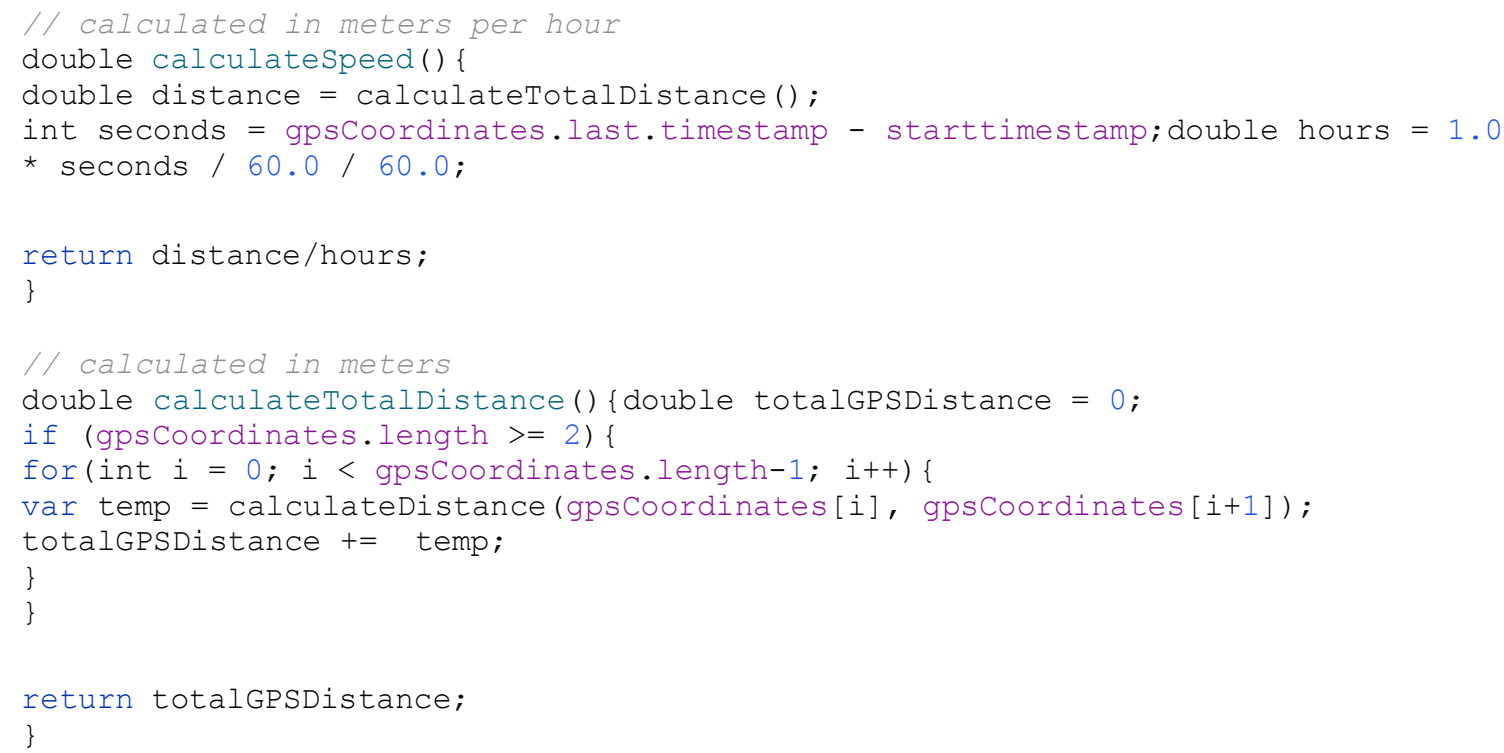




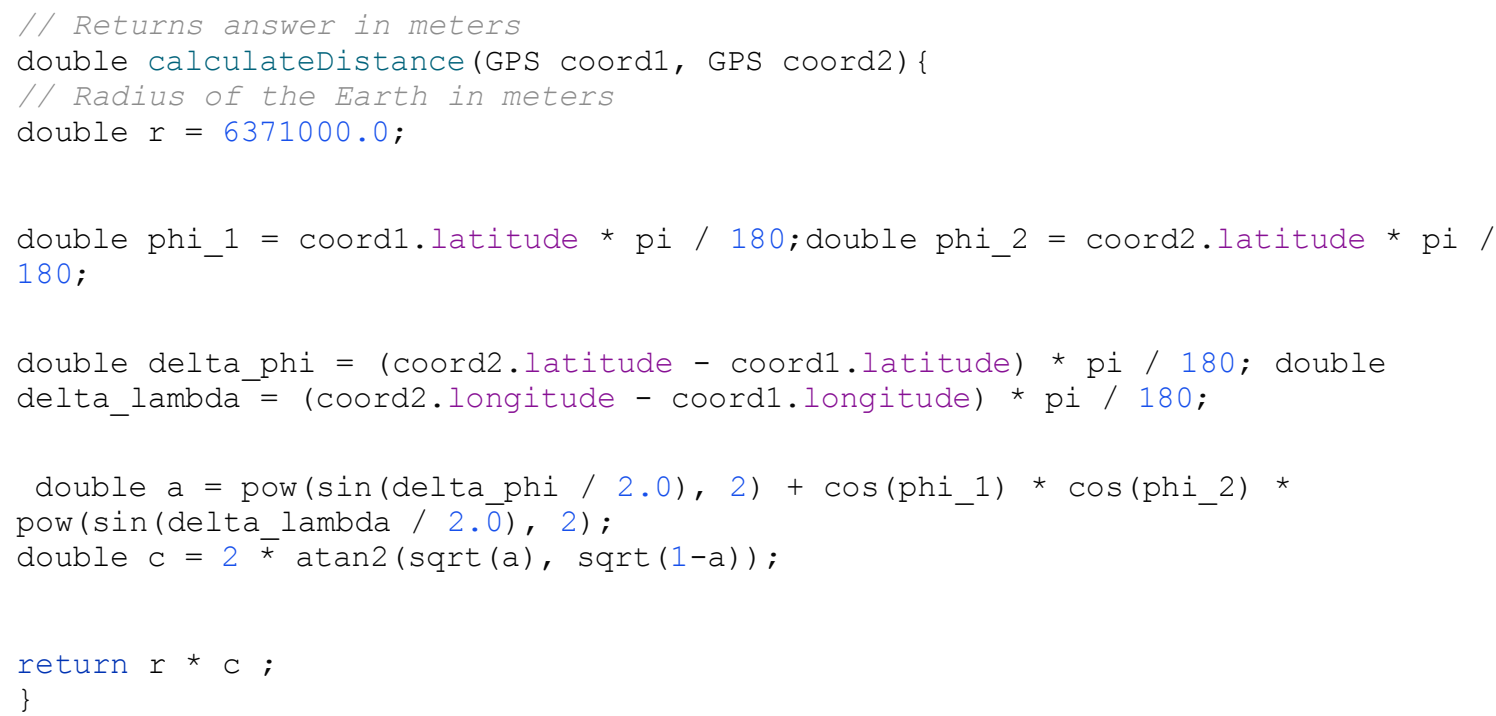

We used a framework called Flutter and it helped us create the inner design of the app and the tracking equipment. It helped us simulate the app on a phone so that we could just make an app without publishing it and using it on the phone.

We later brought in the use of a firebase as well. Firebase helps us keep all the data we use that we get from our hardware. The hardware comes from a company called Particle and it helps us send the data from the tracker with a cellular signal.

\section{EXPERIMENT}

Two experiments have been conducted in order to measure the accuracy and scalability.

\subsection{Experiment 1. The accuracy of detecting if the player crosses the line or not}

The experiment is conducted as follows: one player carries the device and crosses the line 50 times. We will use whether the device detects whether the player crosses the line or not. We use the speed algorithms to help devices determine whether a player crosses the line or not. 
Computer Science \& Information Technology (CS \& IT)

Table 1. same player cross the line for 50 times

\begin{tabular}{|c|c|c|c|}
\hline $\begin{array}{c}\text { same player } \\
\text { cross the line } \\
\text { for } 50 \text { times }\end{array}$ & & & \\
\hline & time & speed $(\mathrm{m} / \mathrm{s})$ & device output \\
\hline & 1 & 3 & cross \\
\hline
\end{tabular}

\begin{tabular}{|l|l|l|l|}
\hline & 2 & 2.5 & cross \\
\hline & 3 & 3.5 & cross \\
\hline
\end{tabular}

\begin{tabular}{|l|l|l|l|}
\hline & 3 & 3.5 & cross \\
\hline & 4 & 3.8 & cross \\
\hline & 5 & 4.2 & cross \\
\hline 6 & 4.5 & cross \\
\hline
\end{tabular}

\begin{tabular}{|l|l|l|l|}
\hline & 7 & 2.1 & cross \\
\hline & 8 & 2.8 & cross \\
\hline & 9 & 3.2 & cross \\
\hline
\end{tabular}

\begin{tabular}{|l|l|l|l|}
\hline & 10 & 4.2 & cross \\
\hline & 11 & 4.3 & cross \\
\hline & 12 & 3.2 & cross \\
\hline & 13 & 4.6 & cross \\
\hline & 14 & 5.8 & \\
\hline
\end{tabular}

\begin{tabular}{|l|l|l|l|}
\hline & 14 & 5.8 & not cross \\
\hline & 15 & 5.4 & cross \\
\hline & 16 & 2.4 & cross \\
\hline & 17 & 2.3 & cross \\
\hline
\end{tabular}

\begin{tabular}{|l|l|l|l|}
\hline & 21 & 4.9 & not cross \\
\hline & 22 & 4.3 & cross \\
\hline & 23 & 4.8 & cross \\
\hline & 24 & 5.1 & cross \\
\hline & 25 & 3.7 & cross \\
\hline & 26 & 3.4 & cross \\
\hline & 27 & 2.9 & cross \\
\hline & 28 & 1.8 & cross \\
\hline & 29 & 3.8 & not cross \\
\hline & 30 & 3.6 & cross \\
\hline & 31 & 4.7 & cross \\
\hline & 32 & 5.4 & cross \\
\hline
\end{tabular}




\begin{tabular}{|c|c|c|}
\hline 33 & 3.9 & cross \\
\hline 34 & 4.3 & cross \\
\hline 35 & 2.2 & cross \\
\hline 36 & 3.8 & cross \\
\hline 37 & 1.3 & cross \\
\hline 38 & 1.5 & cross \\
\hline 39 & 2.1 & cross \\
\hline 40 & 4.4 & cross \\
\hline 41 & 2.8 & cross \\
\hline 42 & 5.3 & not cross \\
\hline 43 & 4.8 & cross \\
\hline 44 & 4.1 & cross \\
\hline 45 & 3.2 & cross \\
\hline 46 & 2.6 & cross \\
\hline 47 & 3.3 & cross \\
\hline 48 & 2.4 & cross \\
\hline 49 & 3.2 & cross \\
\hline 50 & 4.1 & cross \\
\hline
\end{tabular}

Summary: from this data, we can see that the device generally captures whether the player passes the line or not with an accuracy of $92 \%$. However, sometimes it does not capture the data because the player's speed is probably high or the device has margin of error.

\section{Experiment 2. The accuracy of detecting multiple football players crossing line activityconcurrently}

We try to let different football players carry this device and cross lines to find out whether this device works for various situations.

In this experiment, 5 different players will carry this device and cross the line. Each player will use different speeds to do this for 10 times. 
Table 2. different players cross the line

\begin{tabular}{|c|c|c|c|}
\hline \multirow[t]{11}{*}{ player1 } & time & speed $(\mathrm{m} / \mathrm{s})$ & device output \\
\hline & 1 & 3.6 & cross \\
\hline & 2 & 2.8 & cross \\
\hline & 3 & 2.5 & cross \\
\hline & 4 & 3.9 & cross \\
\hline & 5 & 4.3 & cross \\
\hline & 6 & 4.9 & cross \\
\hline & 7 & 5.2 & cross \\
\hline & 8 & 3.3 & cross \\
\hline & 9 & 4.6 & not cross \\
\hline & 10 & 3.7 & cross \\
\hline \multirow[t]{10}{*}{ player 2} & 1 & 3.2 & cross \\
\hline & 2 & 3.9 & cross \\
\hline & 3 & 3.6 & cross \\
\hline & 4 & 2.8 & cross \\
\hline & 5 & 3.4 & cross \\
\hline & 6 & 4.3 & cross \\
\hline & 7 & 4.7 & cross \\
\hline & 8 & 5.1 & cross \\
\hline & 9 & 2.4 & cross \\
\hline & 10 & 3.4 & cross \\
\hline \multicolumn{4}{|l|}{ player3 } \\
\hline & 1 & 2.3 & cross \\
\hline & 2 & 2.8 & cross \\
\hline & 3 & 3.3 & cross \\
\hline & 4 & 3.5 & cross \\
\hline & 5 & 3.1 & cross \\
\hline & 6 & 3.8 & not cross \\
\hline
\end{tabular}




\begin{tabular}{|c|c|c|c|}
\hline & 7 & 4.1 & cross \\
\hline & 8 & 3.4 & cross \\
\hline & 9 & 3.9 & cross \\
\hline & 10 & 3.2 & cross \\
\hline \multirow[t]{10}{*}{ player 4} & 1 & 3.4 & cross \\
\hline & 2 & 3.6 & cross \\
\hline & 3 & 2.7 & cross \\
\hline & 4 & 4.1 & cross \\
\hline & 5 & 4.3 & cross \\
\hline & 6 & 4.6 & cross \\
\hline & 7 & 2.7 & cross \\
\hline & 8 & 3.1 & cross \\
\hline & 9 & 3.5 & cross \\
\hline & 10 & 3.7 & cross \\
\hline \multirow[t]{10}{*}{ player 5} & 1 & 2.8 & cross \\
\hline & 2 & 3.2 & cross \\
\hline & 3 & 3.7 & cross \\
\hline & 4 & 5.1 & cross \\
\hline & 5 & 2.6 & cross \\
\hline & 6 & 4.2 & cross \\
\hline & 7 & 3.3 & not cross \\
\hline & 8 & 3.8 & cross \\
\hline & 9 & 3.1 & cross \\
\hline & 10 & 2.4 & cross \\
\hline
\end{tabular}

From this result, we can find out that the device generally captures players crossing the line precisely with an accuracy of $94 \%$. Few errors (results that show players didn't cross the line) are shown.

From these results above, we can find out that in two experiments, the device successfully detects players crossing the line most times. This shows the device can detect the location properly no matter which player is carrying the device and the speed players run. This shows that the device has relatively high accuracy. The result also meets our expectation that the device will detect most situations in which players cross the line. The evidence is that among hundreds of times players cross the line, there's only fewer than 10 times that the device didn't detect players crossing the line. 


\section{RELATED WORK}

This work titled "Architecture of an IoT-based system for football supervision (IoT Football)" is to use communication technology like ZigBee (one type of personal area networks) and embed sensing devices (e.g. sensors and RFID) [16]. By collecting footballers' information through sensors and sending them to the cloud, this device can help monitor the health of footballers and reduce the occurrence of adverse health conditions like hypoglycemia, swallowing the tongue and shortness of breath.

These two works share some similarities and have quite a difference. The similarity between our program and their program is that both use sensing devices to collect necessary data for certain purposes. Both programs also use communication technology that lets the information be sent from the device to the cloud. One difference between them is that two programs collect different sets of data. Their program collects footballers' health data while our program collects footballers' distance and location data. Their program uses communication technology like Zigbee while our program uses communication technology $3 \mathrm{~g}$ cellular [10].

The clear advantage of our solution is that it can be used easier in a variety of locations. This advantage is caused by $3 \mathrm{~g}$ cellular. Their communication solution using Zigbee is limited in a small scale and needs professionals to debug them. So, our solution with a $3 \mathrm{~g}$ network can be used in numerous locations around the United States and can be used in large areas like football fields.

This work titled "IoT for Next-Generation Racket Sports Training" is to collect information by using wireless wearable sensing device (WSD) [11]. The system of WSD is capable of recognizing three different actions, i.e., smashes , clears , and drops , with an accuracy rate of 97\% [12]. With this information, they can differentiate racket sports players between professional, subelite, and amateur players from their stroke performance. This IoT framework aims to change the way of racket sports training from experience-driven (subjective) to data-driven (objective).

Our work has several similarities and differences between this work. The similarity is that both works use data to provide advice for sports. Same as the WSD of this work, our system is lowcost, easy-to-use, and computationally efficient. The difference is that their solution shows users their level of performance (professional, subelite, and amateur players), while our solution presents distance, speed, and duration time.

Our solution provides users with more detailed information in an intuitive way. By carrying our devices in the football game, users can get detailed information like speed, distance and duration right after the game, they can also see the map so that they know their trajectory in the game. This gives footballers the opportunity to improve their performance with collected information.

This work titled "Continuous health monitoring of sportspeople using IoT devices based wearable technology" is to be used in wearable tracking devices to collect the health details and track the exercise records for reducing the risk factors. Machine learning techniques are introduced to analyze and monitor sportspersons' health.

Our work has several similarities and differences. The similarity is that both works use tracking devices in order to monitor and analyze important data of sportsmen. The difference is that this work uses wearable devices while our work uses dedicated devices that sportsmen carry on. The second difference is that our device tracks data like speed, velocity, and location while this device tracks health-related data. 
Our solution can show you your trajectory during matches and football players can find out the data easily on their mobile phone [15]. They can use this data to further improve their performance and know what they did during matches. Their solution, however, emphasizes on health which sportsmen need professional doctors in order to make the data meaningful. So the threshold for football players to use the data of their device is quite high.

\section{Conclusions}

We will have several people run the same routes on the same field. If everyone runs at a decent pace, we should be getting different samples of data per person. If the tracker can show us the difference between each player and if it is accurate enough to tell us where each player is where the coach needs to know where they are, we can tell that the application is accurate.

Accuracy- Although we use more professional sensors to collect crucial information, it's inevitable that there's error in locations and distance [13]. The problem is that the GPS and the distance counting sensor do not exactly reflect this information. The location and distance we get from the sensor may have a little bit of variation compared to the true location and distance.

Practicability- The price of tracking equipment (Particles tracking one) is a little expensive. It costs about 160 dollars per tracker so there's still room for price reduction to make it more universal, helping more people.

Optimization- We can still improve the process of collecting data so that this process is easier.

In the future, if possible, we could upgrade our current hardware equipment to $4 \mathrm{G}$ devices instead of staying on $3 \mathrm{G}$. I feel like $4 \mathrm{G}$ could improve the signal and perhaps send out more accurate data instead of being just an estimated location. $4 \mathrm{G}$ could also reach out to more rural locations so the technology is more available to everyone. We can also have the tracker run at smaller interval ticks so that the data given will be more accurate.

Currently the tracking device is quite expensive, so in the future if one of the companies decide to sponsor us, we could use that money to make the device a lot less expensive [14]. Thus more teams could use our equipment at a lower price and not have to worry about it being worse in quality.

\section{REFERENCES}

[1] Lee, In, and Kyoochun Lee. "The Internet of Things (IoT): Applications, investments, andchallenges for enterprises." Business Horizons 58.4 (2015): 431-440.

[2] Ishida, Kazunari. "IoT application in sports to support skill acquisition and improvement." 2019IEEE 12th Conference on Service-Oriented Computing and Applications (SOCA). IEEE, 2019.

[3] Wang, Yufan, et al. "IoT for next-generation racket sports training." IEEE Internet of Things Journal 5.6 (2018): 4558-4566.

[4] Huifeng, Wang, Seifedine Nimer Kadry, and Ebin Deni Raj. "Continuous health monitoring of sportsperson using IoT devices based wearable technology." Computer Communications 160 (2020): 588-595.

[5] Madakam, Somayya, et al. "Internet of Things (IoT): A literature review." Journal of Computer and Communications 3.05 (2015): 164.

[6] Ikram, Mohammed Abdulaziz, Mohammad Dahman Alshehri, and Farookh Khadeer Hussain. "Architecture of an IoT-based system for football supervision (IoT Football)." 2015 IEEE 2nd World Forum on Internet of Things (WF-IoT). IEEE, 2015.

[7] Nastic, Stefan, et al. "Provisioning software-defined IoT cloud systems." 2014 internationalconference on future internet of things and cloud. IEEE, 2014.

[8] Gerhana, Yana Aditia, et al. "Decision support system for football player's position with tsukamoto fuzzy inference system." MATEC Web of Conferences. Vol. 197. EDP Sciences, 2018. 
[9] Bi, Zhuming, et al. "IoT-based system for communication and coordination of football robot team." Internet Research (2017).

[10] Markov, Marko, et al. "Application of Firebase Cloud Service for Storing and Analyzing Data from IoT Mobile Devices." J. Mech. Autom. Identif. Technol 3 (2018): 17-20.

[11] Xu, Yetong, and Wenwu Hu. "Load evaluation of campus football match based on microprocessorand IoT wearable equipment." Microprocessors and Microsystems 81 (2021): 103778.

[12] Yu, F. U. "IoT Application in Monitoring System of Competitive Sports." Communications Technology (2012)

[13] Farooq, M. Umar, et al. "A review on internet of things (IoT)." International journal of computer applications 113.1 (2015): 1-7.

[14] Wilkerson, Gary B., Ashish Gupta, and Marisa A. Colston. "Mitigating sports injury risks using internet of things and analytics approaches." Risk analysis 38.7 (2018): 1348-1360.

[15] Elijah, Olakunle, et al. "An overview of Internet of Things (IoT) and data analytics in agriculture: Benefits and challenges." IEEE Internet of Things Journal 5.5 (2018): 3758-3773.

[16] Stoyanova, Maria, et al. "A survey on the internet of things (IoT) forensics: challenges, approaches, and open issues." IEEE Communications Surveys \& Tutorials 22.2 (2020): 1191-1221.

(C) 2021 By AIRCC Publishing Corporation. This article is published under the Creative Commons Attribution (CC BY) license. 